\title{
RF- Energy Harvesting (RF-EH) for Sustainable Ultra Dense Green Network (SUDGN) in 5G Green Communication
}

\author{
Devasis Pradhan ", IEEE Member, Priyanka K C, IEEE Member
}

Assistant Professor, Department of Electronics \& Communication Engineering, Acharya Institute of Technology, Dr. Sarvepalli Radha Krishan Road, Bengaluru, India

\author{
DOI: $10.36348 /$ sjet.2020.v05i06.001 \\ | Received: 26.05.2020 | Accepted: 07.06.2020 | Published: 11.06.2020 \\ *Corresponding author: Devasis Pradhan
}

\section{Abstract}

5G Network strongly depend upon ultra dense integrated small cell beside macro cell. This kind of UDN consists of large number of macro cell and small cells through which increase in energy demands can be met. Practically energy consumption is done by dynamically controlling modes in radio networks. This paper reflects the significant power saving by considering only a subset of network node which reduce traffic migration and control phase signaling. To enable the sustainable UDGN improve system energy efficiency, and alternative energy source are introduce to reduce dependence on traditional fossil fuels. In 5G optimizing energy is the most important challenge and maintenance of RF communication system. RF energy harvesting is a unique technique for $5 \mathrm{G}$ system as an alternative to traditional energy supply sources. Future evolution of the networks has also been assumed to open many new business horizons for the operators and the need of not only a resource efficient but also an energy efficient ecosystem has greatly been felt. The deployment of small cells has been envisioned as a promising answer for handling the massive heterogeneous traffic, but the adverse economic and environmental impacts cannot be neglected.

Keywords: 5G; energy-efficiency; wireless; sustainable; energy harvesting; green technology; renewable energy; ultradense network.

Copyright @ 2020: This is an open-access article distributed under the terms of the Creative Commons Attribution license which permits unrestricted use, distribution, and reproduction in any medium for non-commercial use (NonCommercial, or CC-BY-NC) provided the original author and source are credited.

\section{INTRODUCTION}

In order to meet the requirements of next generation networks are to support hundreds of millions of connected devices, 10 to 100 times higher user data rate, and 10 times longer battery life at similar cost and energy dissipation as of today. The integration of the new radio concepts such as Massive Multi-Input Multi-Output (MIMO), Ultra dense green network (UDGN), device to device (D2D) Communication, Ultra-Reliable Communication, Massive Machine Type Communication (MTC) and the exploitation of new spectrum bands will allow supporting these huge demands.

Considering energy and spectrum are the most important resources in mobile communication, energy consumption however is growing significantly. The Information and Communication Technology (ICT) field is responsible for about $3 \%$ of energy consumption and $2 \%-4 \%$ of $\mathrm{CO}_{2}$ emissions in the world. Energy consumption is increasing at a rate of $15 \%-20 \%$ annually which means double consumption after about 5 years. Base Stations (BSs) consume $60 \%-80 \%$ of the total energy. These expectations and figures motivate more research on energy management especially in $5 \mathrm{G}$ UDGN towards green communication.

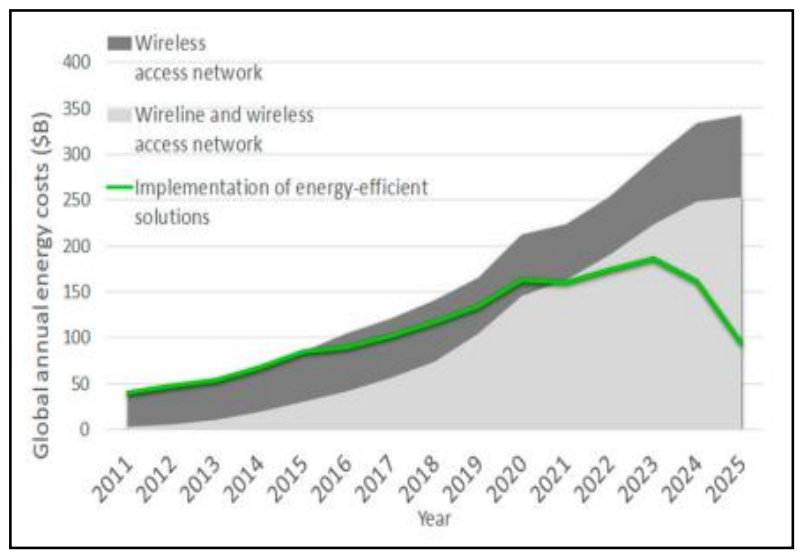

Fig-1(a): Estimation of costs for the global annual energy consumption of telecommunication networks in period 2011-2025 


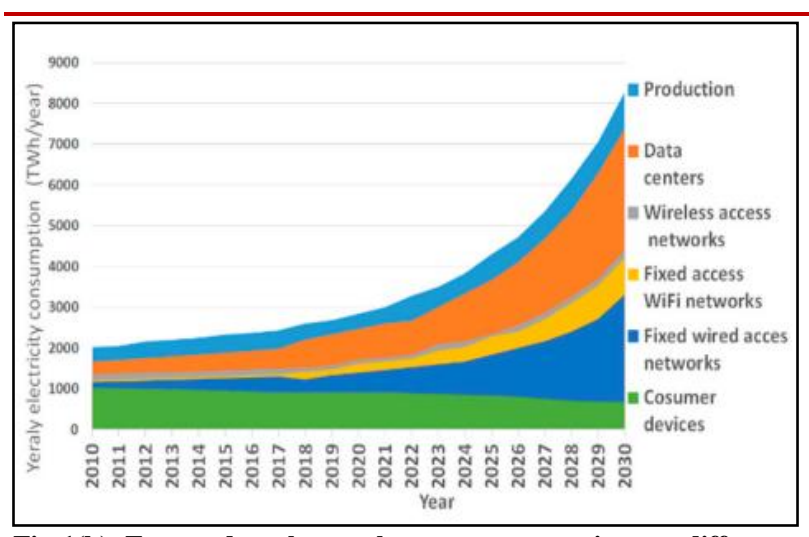

Fig-1(b): Expected total annual energy consumption per different ICT systems in period 2010-2030

High energy costs of telecommunication networks presented in Figure 1a correlate with estimations of energy consumption trends of different ICT systems presented in Figure 1b. Estimates presented for the period 2011-2030 are performed with an assumption that takes into account expected annual: future improvements in the energy efficiency of ICTs systems, trends in future IP traffic growth and future improvements in electricity usage.

Data rates as high as that of 1 Gbps have been foreseen with the advent of $5 \mathrm{G}$. In addition, with an explosive number of heterogeneous devices coming online, including sensors for home security, tablets, and wearable health monitors, the computational power of base stations must increase. In 5G small cells are inevitable in deployments due to their advantage of improved traffic handling within a smaller area as well as the shorter cell ranges that result from the use of higher frequencies. Yet, the increasing number of base stations translates into more energy consumption, although the increase in consumption will not be linear. Small cells, or in other words densification, call for sophisticated management of resources.

Most recently, intelligent resource allocation and control techniques utilizing machine learning algorithms have been suggested to help next generation radios in their autonomous reconfiguration for improving the data rates, energy efficiency and interference mitigation. This paper gives an overview of Energy Harvesting (EH) techniques, in particular on EH power management policies optimizations, with specific stress on Wireless Sensor Networks (WSNs) and on ambient RF- Energy Harvesting (RF-EH).

\section{Overview of 5 G Network}

The 5G networks stand for fifth generation mobile technology and can outperform earlier versions of wireless communication technology. The new technology provides diverse abilities and encourages full networking among countries globally. Accordingly, $5 \mathrm{G}$ architecture constitutes both licensed and unlicensed frequency bands. In a recent study from 2016, the Federal Communications Commission's (FEC) announced the use-case of $60 \mathrm{GHz}$ spectrum with the range of $57 \mathrm{GHz}-71 \mathrm{GHz}$ for the unlicensed wireless category. These networks are expected to implement very high service quality. In order to entertain these $5 \mathrm{G}$ services for the new generation communication system, different new technologies have been proposed, namely Millimeter wave communication, HetNet (Heterogeneous Networks), Massive multiple-input multiple-output (MIMO), and visual light communication.

Consequently, even with the mentioned $5 \mathrm{G}$ paradigms for improving EE, the power consumption will still grow because of the explosive data rate requirements in the future. Therefore, improving EE can only alleviate the power consumption problem to a certain extent and is insufficient for enabling sustainable $5 \mathrm{G}$ communications. By energy harvesting technologies, which allow BSs and devices to harvest energy from renewable resources (solar, wind, etc.) and even RF signals (television signals, interference signals, etc.), have received significant attention recently. They provide green energy supply solutions for powering different components of wireless communication networks and named as Green Network. This paper focuses on the state of the art of green $5 \mathrm{G}$ and $\mathrm{RF}$ energy harvesting technologies, which will create an ecosystem of $5 \mathrm{G}$ wireless networks as shown in Figure. 2

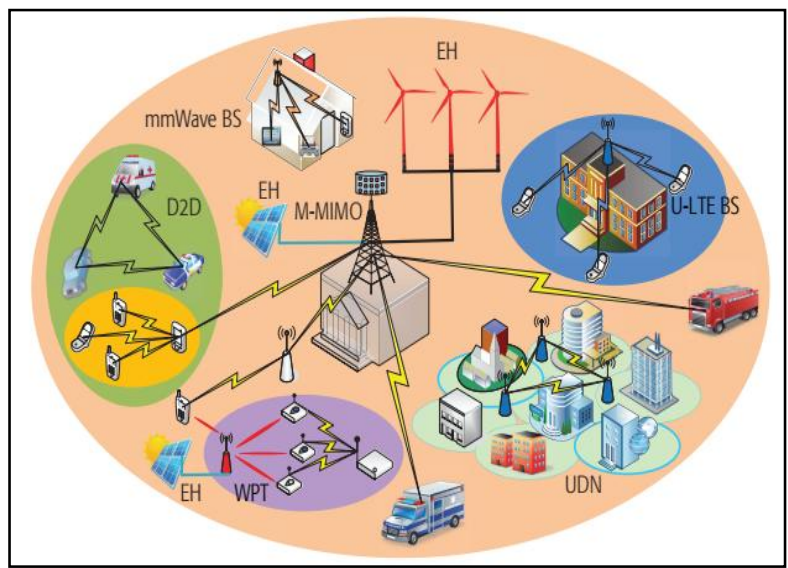

Fig-2: Ecosystem of 5G Wireless Networks

\section{G Wireless Network- Architecture}

The 5G networks for future applications in all domains provide prospects for a fully connected society, for all connectivity between the devices provides a broader range of new business structures, which subsequently paves a path towards different industry profiles, such as energy and manufacturing sectors. In accordance with this, 5G shall provide subsequent connections between different analyses for human-to-machine and machine-to-machine interactions. The simultaneous co-existence with human and machine interactions shall provide different performance indications where a $5 \mathrm{G}$ network can provide magnificent support. There is a need to produce 
Devasis Pradhan \& Priyanka K C., Saudi J Eng Technol, June, 2020; 5(6): 258-264

suitable architectures with slicing networks to satisfy the present day telecommunication scenario. The table 1 discussed the comparison of $5 \mathrm{G}$ technology architecture.

Table-1: Different 5G communication techniques

\begin{tabular}{|c|c|c|}
\hline Architecture & Pro's & Con's \\
\hline $\begin{array}{l}\text { Mm-Wave - a mm Wave Base station (MBS) is } \\
\text { considered to be in a highertier, and small base } \\
\text { stations work under the control of MBS. }\end{array}$ & $\begin{array}{l}\text { The major advantages are better and higher data } \\
\text { rates with considerable reduction of energy } \\
\text { consumed. Among the MBS, less congestion is } \\
\text { made and easy hand-off is attainable. }\end{array}$ & $\begin{array}{l}\text { Low reliability and } \\
\text { comparatively very high } \\
\text { operational cost between } \\
\text { the MBS. }\end{array}$ \\
\hline $\begin{array}{l}\text { C-RN (Cognitive Radio Network) }- \text { In this the } \\
\text { base cell stations are more cognitive with } \\
\text { cognitive radio nodes of secondary users. The } \\
\text { main licensed users are in the primary nodes in } \\
\text { function. }\end{array}$ & $\begin{array}{l}\text { The major pros of this structure are minimum } \\
\text { interference and improved network capacity } \\
\text { with respect to higher bandwidth coverage and } \\
\text { data rate perusal. }\end{array}$ & $\begin{array}{l}\text { Less energy efficiency and } \\
\text { a major trade-off between } \\
\text { the spatial frequency and } \\
\text { the range of outage. }\end{array}$ \\
\hline $\begin{array}{l}\text { D2D Communication - With less involvement } \\
\text { of MBS, this allows the user equipment to } \\
\text { communicate efficiently }\end{array}$ & $\begin{array}{l}\text { Reliable links that provide high data rate and } \\
\text { instant communication with quick file sharing. }\end{array}$ & $\begin{array}{l}\text { Secure communication } \\
\text { must be provided with } \\
\text { proper link }\end{array}$ \\
\hline $\begin{array}{l}\text { LTE-U - LTE-U further increases the LTE } \\
\text { capacity by enabling the LTE air interface to } \\
\text { operate in unlicensed spectrum. The unlicensed } \\
\text { spectrum available in the } 5 \mathrm{GHz} \text { band/WiFi band } \\
\text { ( } \geq 400 \mathrm{MHz} \text { bandwidth) provides LTE-U with } \\
\text { additional spectrum resources. }\end{array}$ & $\begin{array}{l}\text { LTE employs a scheduling-based channel } \\
\text { access mechanism, where } \\
\text { multiple users can be served simultaneously by } \\
\text { assigning them different resource blocks. }\end{array}$ & $\begin{array}{l}\text { LTE-U- transmission may } \\
\text { generate continuous } \\
\text { interference to WiFi } \\
\text { systems such that the } \\
\text { channel is detected as busy } \\
\text { most of the time. }\end{array}$ \\
\hline $\begin{array}{l}\text { M-MIMO - M-MIMO is expected to provide at } \\
\text { least an order of magnitude improvement in } \\
\text { multiplexing gain and array gain via deploying a } \\
\text { large number of antennas at the BSs while } \\
\text { serving a large number of users with the same } \\
\text { time-frequency resource. }\end{array}$ & $\begin{array}{l}\text { Transmitted power largely dominates the } \\
\text { overall power consumption, deploying more } \\
\text { antennas yields a higher Energy Efficient. }\end{array}$ & $\begin{array}{l}\text { System performance } \\
\text { bottleneck due to more } \\
\text { stringent requirements on } \\
\text { power consumption and } \\
\text { limited physical size. }\end{array}$ \\
\hline $\begin{array}{l}\text { UDNs- UDNs are obtained by extensively } \\
\text { deploying diverse types of BSs in hotspot areas } \\
\text { and can be regarded as a massive version of } \\
\text { heterogeneous networks }\end{array}$ & $\begin{array}{l}\text { Signaling and data as well as uplink and } \\
\text { downlink transmissions are decoupled. } \\
\text { Decoupling of signaling and data is the hyper- } \\
\text { cellular structure where macrocells provide } \\
\text { umbrella coverage, and microcells (utilizing, } \\
\text { e.g., mmWave BSs) aim to fulfill the high } \\
\text { capacity demands. }\end{array}$ & $\begin{array}{l}\text { Completely eliminating } \\
\text { interference is overly } \\
\text { optimistic for practical } \\
\text { systems, it is expected that } \\
\text { removing the two or three } \\
\text { strongest interferers }\end{array}$ \\
\hline
\end{tabular}

\section{Energy Efficiency of 5 G System}

In order to increase the coverage and the capacity of the system, power consumption is expected to increase at the same time, a challenging issue for $5 \mathrm{G}$. Optimizations, categorized on green energy researches, aim BS energy use reduction. In fact, the power consumption in the Operation Expenditure (OPEX) in today's mobile systems is dominated by networks, up to $90 \%$, and only $10 \%$ is for mobile user. The network electricity supply is thus a critical issue in mobile industry, especially for regions with difficult grid power connection. Alternative energy sources, light, wind, vibrations, etc. are considered. RF-EH appears as a candidate technology to further optimize energy management in 5G. In particular, EH from RF signals are actively studied and mathematical models are made in order to make it possible in the near future. $\mathrm{EH}$ relays harvest energy from the BS transmissions following two power splitting strategies at the relays for $\mathrm{EH}$, and analytical expressions of outage probability are given for these strategies.

The interface between network and terminal can use massive MIMO technique to enhance EE, in addition to new waveforms and to network radio resources optimization. The air interface, procedures, and signaling can also be enhanced in terms of energy consumption through sleep modes, simplified procedures access schemes, and more efficient modulation, spreading and coding that allow low transmission powers. Energy consumption can be reduced using EH from environmental energy sources, such as light, heat and vibration.

\section{Consumption of Energy by Users}

The energy consumption patterns of userrelated devices point to different challenges and require different approaches to energy consumption reductions. Energy consumption estimates of user-related devices for the period 2011-2025 are presented in Figure 3. Presented estimates are performed for all connected user devices in cellular networks, internet of things (IoT) applications, public safety, and intelligent buildings and generally for all consumer devices with a network connection. Estimates consider the explosive growth of user-related devices from about 50 billion in 2011 to 110 billion devices connected to the network in 2025. Forecasts for the global energy consumption of these user-related devices estimate the energy consumption raise from about $180 \mathrm{TWh}$ in 2011 to $1400 \mathrm{TWh}$ in 2025 (Figure 3), which represents a 7.7 time increase in the period of one and a half-decade. 
Devasis Pradhan \& Priyanka K C., Saudi J Eng Technol, June, 2020; 5(6): 258-264

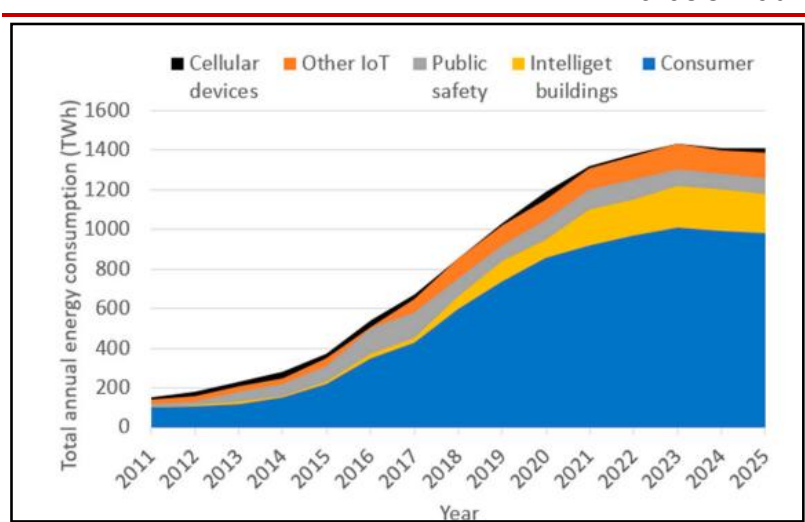

Fig-3: Expected energy consumption of devices for the period 2011-2025

For 5G networks, as currently the most prominent wireless network technology, tremendous performance improvements are envisioned. These improvements encompass support of: a thousand-fold increase in throughput in comparison to present networks, up to ca. 7.6 billion mobile subscribers with the connection of at least 100 billion devices worldwide, up to $10 \mathrm{~Gb} / \mathrm{s}$ individual user broadband speeds, IoT communications, tactile Internet applications and the network latency of $1 \mathrm{~ms}$ or lower.

\section{Renewable Energy Sources}

Based on powering Base Stations (BSs) sites using energy harvested from renewable energy sources such as wind, solar, fuel cell or combination of these energy sources significantly contributes to the improvement of wireless network Energy Efficient. Current trends in terms of integration of renewable energy into power supply systems of contemporary wireless networks are twofold. The first analogy is dedicated to the replacement of an off-grid diesel-based BS power supply system with those relying solely on some renewable energy sources. The other analogy is based on the so-called hybrid BSs sites which use different renewable energy sources or a mix of renewable, diesel generator and/or grid energy. In addition to EE improvements and operational expenditure reductions, such approaches significantly reduce or even completely eliminate diesel generator $\mathrm{CO}_{2}$ emissions from BS sites.

The integration of renewable energy sources into BSs power supply systems can provide compensation for the additional circuit power consumption in case of installing more BSs on BS site. Also, dense allocation of BSs employing energy harvesting from renewable sources can facilitate possible energy cooperation between BSs. This cooperation can be based on transferring through power lines superfluous energy collected on sites harvesting more energy, to BSs sites that harvest less energy.

The major challenge in realization of durable BS site power supply solutions can be found in the intermittent nature of renewable energy sources, limited battery capacities installed on sites and necessity for ensuring stable and without any interruptions power supply of BSs sites. This imposes the development of resource allocation algorithms for the management of BS site power demand. Such algorithms must consider the traffic variations and wireless channel state information0s, power supply impacted with the unpredictable nature of renewable energy sources and battery recharging and depletion cycles.

\section{RF- Energy Harvesting}

$\mathrm{RF}$ energy harvesting, allows small receivers which are expected to be massively used in $5 \mathrm{G}$ use cases like IoT to harvest energy from RF signals which will be received. It is assumed to be a promising technology for powering a huge number of devices, since harvested energy from RF eliminates the need for powering those devices from an electric gird and also enables battery lifetime extension of mobile, sensor or actuator devices. Although RF energy can be fully managed at the receiver side, in the practical implementation of RF energy harvesting, the main challenge is ensuring optimal balance among the harvested energy and the achievable transmission rates. This balance can be realized through the implementation of an approach based on exploiting simultaneous wireless information and power transfer, where the receiver device during reception divides the received signal into two parts, one for energy supply obtained through energy harvesting and the other for information decoding.

RF energy harvesting brings many advantages; the major implementation issue is system performance which is significantly limited by the severe RF signal path loss and consequently low energy conversion efficiency at the position of the energy harvester. One of the possible approaches to system performance improvement is in the implementation of an energy beam-forming concept. This concept is based on the transmission of narrow beams through multiple antennas with optimized beam-forming vectors, which is fully compatible with M-MIMO and $\mathrm{mm}$ Wave theologies. Moreover, D2D communications and ultradense networks (UDN) are technologies that contribute to performance improvements of energy harvesting systems.

\section{Enhancement of RF Energy toward Green Network}

The main advantages of RF Energy harvesting, the system performance is basically constrained by the low energy conversion efficiency and the severe path loss during RF transmission. Generally, the 5G techniques that are able to improve the performance of a wireless communication link can also are exploited to improve the efficiency of RF-EH. For example, narrow beams can be realized by employing multiple antennas at the transmitter side with optimized beam-forming vectors, which is known as energy beam-forming, and this fits the applications of M-MIMO and mm-Wave 
well. In addition to that short-range-communicationbased $5 \mathrm{G}$ techniques such as D2D communications and UDNs are capable of improving the efficiency of RFEH by reducing the energy transfer distance. Besides improving the amount of harvested energy, the circuit power consumed by information decoding for simultaneous wireless information and power transfer is also an important design issue since it reduces the net harvested energy that can be stored in the battery for future use. In particular, the active mixers used in conventional information receivers for RF to baseband conversion are substantially power-consuming. This contributes to form a UDGN which is ultra dense green network as shown in Figure 4.
The most important part of RF-EH is dedicated co-channel interference signals can be exploited for energy harvesting. This fundamentally shifts our traditional viewpoint of "harmful" co-channel interference, which now becomes a potential source of energy. In this regard, UDNs and D2D underlaying communications, where the spectrum is heavily reused across the whole network, provide plentiful opportunities for RF energy to extract the potential gain from co-channel interference. In practice, RF energy enabled devices can harvest energy when co-channel interference is strong and decode information when cochannel interference is relatively weak.

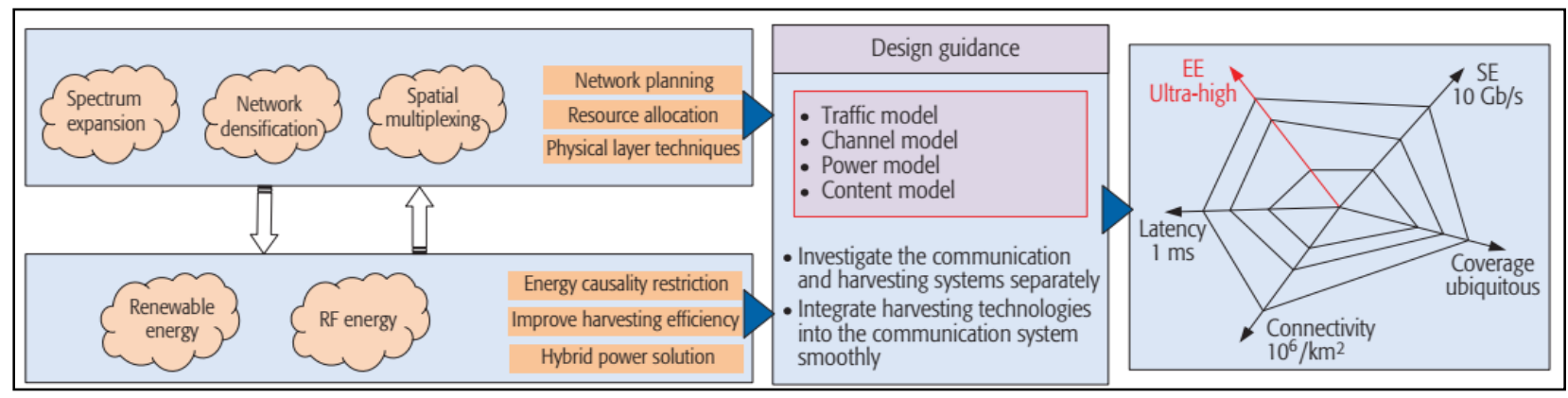

Figure4. Component of Sustainable Ultra Dense Green Network (UDGN)

\section{Frame work of RF-Energy Efficiency (RF-EE) in 5G Network}

The evolution towards self-organized and sustainable 5G cellular networks, coupled with innovative technologies such as network softwarization, virtualization and network slicing, further adds to the already existing network challenges towards RF-EE improvement. Therefore, it is necessary to define RFEE control framework for identifying and describing the key common RF- EE control functions and the control sequences, and procedures for controlling and managing RF-EE. The framework for RF- EE consists of the following modules:

a) Management of Framework(MRF-EE) Basically it monitors, collects, processes, stores and provides RF-EE related information and statistics including the profiles for traffic, operating conditions, the corresponding achievable RF-EE, the variations of QoS/QoE in reference to the predefined QoS/QoE requirements from the embedded energy metering functions in each of theRF- EE optimization entities. The information is assessed and then sent to the RF-EE control and coordination for adjusting the power saving control operations and possible policy adjustment by the RF-EE policy control.

b) RF-EE Control and Coordination (RF-EECC) In this module defines the automation processes for system control and coordination across all relevant network elements. This function involves monitoring and reporting of the operating conditions such as traffic load and density, operational conditions such as temperature and humidity. The RF-EE optimization entity can be a logical or physical component to execute the $\mathrm{EE}$ policies and the corresponding energy consumption optimization operations.

c) Management Policy (RF-EEMP)-In this module defines and manages the RF-EE control policies related to the energy consumption status and control operations at the network, equipment and site levels. It translates the policy information into configurations at the EE optimization entities. The policy may be adjusted accordingly to achievable RF-EE and the variations of QoS) / QoE.

\section{Requirement for RF-EH}

The RF- EH requirements for the $5 \mathrm{G}$ system state that the $5 \mathrm{G}$ access network shall support an energy saving mode with the following characteristics: The energy saving mode can be activated/deactivated either manually or automatically and the service can be restricted to a group of users (e.g., public safety user, emergency callers). The control systems taking care of cell change for individual users need to design in such a way that the becoming switch-off is known well before it takes place and all the needed procedure related to cell change will be executed.

\section{RF Energy Harvesting Usage \& Consumption}

Device is powered by EH directly, without using any buffer. Data transmission is thus possible only when harvested energy can cover the processing energy cost. Storage device, typically a battery, is used to gather harvested energy. This energy is then 
available for use only in the next time slot and two energy storage devices: a super-capacitor storing the harvested energy for immediate use, and a buffer storing the remaining energy for later use. The major implementation issue is system performance which is significantly limited by the severe RF signal path loss and consequently low energy conversion efficiency at the position of the energy harvester. One of the possible approaches to system performance improvement is in the implementation of an energy beam-forming concept. This concept is based on the transmission of narrow beams through multiple antennas with optimized beam-forming vectors, which is fully compatible with M-MIMO and mm-Wave theologies. Moreover D2D communications and ultra-dense networks (UDN) are technologies that contribute to performance improvements of energy harvesting systems. Since $5 \mathrm{G}$ networks are characterized with very dynamic traffic variations, results of offline algorithms often serve as performance upper bounds for online algorithms.

\section{Emerging techniques and new energy models}

In addition, new emerging technologies can also be used for energy-efficient purposes. In particular, caching and mobile computing have shown significant potential as far as reducing energy consumption is concerned. By an intelligent distribution of frequently accessed content over the network nodes, caching alleviates the need for backhaul transmissions, which results in relevant energy consumption reductions. Instead, mobile computing does not directly reduce the energy consumption, but, similarly to wireless power transfer, it can prolong the lifetime of nodes that are low on battery energy.

Nevertheless, in order to conclusively quantify the impact of these techniques on energy efficiency it is necessary to develop new energy consumption models which take into account the energy consumption associated with overhead transmissions over the backhaul, to feedback signaling, and to the execution of computing operations in digital signal processors.

\section{CONCLUSION}

In this paper, we have provided an overview of some emerging technologies which may make up future $5 \mathrm{G}-$ Green Networks. It has also described technologies to be used for $5 \mathrm{G}$ communication. Energy harvesting underpins the green expectation of $5 \mathrm{G}$ networks, while promising spectrum-efficient $5 \mathrm{G}$ technologies can be tailored to realize energy-efficient wireless networks. Facing the highly diversified communication scenarios of the future, user traffic, channel, power consumption, and even content popularity models need to be taken into account jointly for improving the system RF-EH. This study also discusses the process of the needs of a $\mathrm{EH}$ with $5 \mathrm{G}$ and the areas where the network analysis can be made. Additionally, estimations and analyses of energy costs and $\mathrm{CO}_{2}$ emissions for different ICT systems in the period ranging up to the year 2030 are surveyed. Presented analyses confirm that ICT systems are at a critical point regarding current and future energy consumption of telecommunication networks, Data Centers (DCs) and user-related devices.

\section{REFERENCE}

1. Gunduz, D., Stamatiou, K., Michelusi, N., \& Zorzi, M. (2014). Designing intelligent energy harvesting communication systems. IEEE communications magazine, 52(1), 210-216.

2. Han, S., Chih-Lin, I., Xu, Z., \& Rowell, C. (2015). Large-scale antenna systems with hybrid analog and digital beamforming for millimeter wave 5G. IEEE Communications Magazine, 53(1), 186194.

3. Zeng, Y., \& Zhang, R. (2016). Millimeter wave MIMO with lens antenna array: A new path division multiplexing paradigm. IEEE Transactions on Communications, 64(4), 1557-1571.

4. Zhang, R., Wang, M., Cai, L. X., Zheng, Z., Shen, X., \& Xie, L. L. (2015). LTE-unlicensed: The future of spectrum aggregation for cellular networks. IEEE Wireless Communications, 22(3), 150-159.

5. Samarakoon, S., Bennis, M., Saad, W., Debbah, M., \& Latva-Aho, M. (2016). Ultra-dense small cell networks: Turning density into energy efficiency. IEEE Journal on Selected Areas in Communications, 34(5), 1267-1280.

6. Farhangi, H. (2010). The path of the smart grid. Power and energy magazine, IEEE, 8(1), 1828.

7. Tuballa, M. L., \& Abundo, M. L. (2016). A review of the development of Smart Grid technologies. Renewable and Sustainable Energy Reviews, 59, 710-725.

8. Kabalci, Y. (2016). A survey on smart metering and smart grid communication. Renewable and Sustainable Energy Reviews, 57, 302-318.

9. Ma, K., Liu, X., Liu, Z., Chen, C., Liang, H., \& Guan, X. (2017). Cooperative relaying strategies for smart grid communications: Bargaining models and solutions. IEEE Internet of Things Journal, 4(6), 2315-2325.

10. Yan, Y., Qian, Y., Sharif, H., \& Tipper, D. (2012). A survey on smart grid communication infrastructures: Motivations, requirements and challenges. IEEE communications surveys \& tutorials, 15(1), 5-20.

11. Gungor, V. C., Sahin, D., Kocak, T., Ergut, S., Buccella, C., Cecati, C., \& Hancke, G. P. (2011). Smart grid technologies: Communication technologies and standards. IEEE transactions on Industrial informatics, 7(4), 529-539.

12. Lambert, S., Van Heddeghem, W., Vereecken, W., Lannoo, B., Colle, D., \& Pickavet, M. (2012). Worldwide electricity consumption of communication networks. Optics express, 20(26), B513-B524. 
Devasis Pradhan \& Priyanka K C., Saudi J Eng Technol, June, 2020; 5(6): 258-264

13. Wu, Q., Li, G. Y., Chen, W., Ng, D. W. K., \& Schober, R. (2017). An overview of sustainable green $5 \mathrm{G}$ networks. IEEE Wireless Communications, 24(4), 72-80.

14. Lorincz, J., \& Matijevic, T. (2014). Energyefficiency analyses of heterogeneous macro and micro base station sites. Computers \& Electrical Engineering, 40(2), 330-349.

15. Samarakoon, S., Bennis, M., Saad, W., Debbah, M., \& Latva-Aho, M. (2016). Ultra-dense small cell networks: Turning density into energy efficiency. IEEE Journal on Selected Areas in Communications, 34(5), 1267-1280.

16. Björnson, E., Sanguinetti, L., Hoydis, J., \& Debbah, M. (2015). Optimal design of energyefficient multi-user MIMO systems: Is massive MIMO the answer?. IEEE Transactions on Wireless Communications, 14(6), 3059-3075.

17. Jain, P. P., Pawar, P. R., Patil, P., \& Pradhan, D. (2019). Narrowband Spectrum Sensing in Cognitive Radio: Detection Methodologies.

18. Hu, Feng, Bing Chen, and Kun Zhu. "Full spectrum sharing in cognitive radio networks toward 5G: A survey." IEEE Access 6 (2018): 15754-15776.

19. Mowla, M. M., Ahmad, I., Habibi, D., \& Phung, Q. V. (2017, January). An energy efficient resource management and planning system for $5 \mathrm{G}$ networks. In 2017 14th IEEE Annual Consumer Communications \& Networking Conference (CCNC) (pp. 216-224). IEEE.

20. Ao, W. C., \& Psounis, K. (2017). Approximation algorithms for online user association in multi-tier multi-cell mobile networks. IEEE/ACM

Transactions on Networking, 25(4), 2361-2374.

21. Celebi, H., Yapıcı, Y., Güvenç, I., \& Schulzrinne, H. (2019). Load-based on/off scheduling for energy-efficient delay-tolerant $5 \mathrm{~g}$ networks. IEEE Transactions on Green Communications and Networking, 3(4), 955-970.

22. Labidi, W., Chahed, T., \& Elayoubi, S. E. (2018, September). Battery management for demandresponse in mobile networks powered by a smart grid. In 2018 IEEE 29th Annual International Symposium on Personal, Indoor and Mobile Radio Communications (PIMRC) (pp. 1-7). IEEE.

23. Masoudi, M., Khamidehi, B., \& Cavdar, C. (2017, March). Green cloud computing for multi cell networks. In 2017 IEEE Wireless Communications and Networking Conference (WCNC) (pp. 1-6). IEEE.

24. ETSI ES 203228 V1.2.1.(2017). "Environmental Engineering (EE); Assessment of mobile network energy efficiency - Annex B: Cloud RAN energy efficiency," Standard

25. Silva, P., Almeida, N. T., \& Campos, R. (2019, April). Energy consumption management for dense Wi-Fi networks. In 2019 Wireless Days (WD) (pp. 1-8). IEEE.

26. Beyranvand, H., Lévesque, M., Maier, M., Salehi, J. A., Verikoukis, C., \& Tipper, D. (2016). Toward 5G: FiWi enhanced LTE-A HetNets with reliable low-latency fiber backhaul sharing and $\mathrm{WiFi}$ offloading. IEEE/ACM Transactions on Networking, 25(2), 690-707. 